\title{
Noncrossing partitions and the shard intersection order*
}

\author{
Nathan Reading \\ Department of Mathematics, North Carolina State University, Raleigh, North Carolina 27695-8205, USA
}

\begin{abstract}
We define a new lattice structure $(W, \preceq)$ on the elements of a finite Coxeter group $W$. This lattice, called the shard intersection order, is weaker than the weak order and has the noncrossing partition lattice $\mathrm{NC}(W)$ as a sublattice. The new construction of $\mathrm{NC}(W)$ yields a new proof that $\mathrm{NC}(W)$ is a lattice. The shard intersection order is graded and its rank generating function is the $W$-Eulerian polynomial. Many order-theoretic properties of ( $W, \preceq)$, like Möbius number, number of maximal chains, etc., are exactly analogous to the corresponding properties of $\mathrm{NC}(W)$. There is a natural dimension-preserving bijection between simplices in the order complex of $(W, \preceq)$ (i.e. chains in $(W, \preceq)$ ) and simplices in a certain pulling triangulation of the $W$-permutohedron. Restricting the bijection to the order complex of $\mathrm{NC}(W)$ yields a bijection to simplices in a pulling triangulation of the $W$-associahedron.
\end{abstract}

The lattice $(W, \preceq)$ is defined indirectly via the polyhedral geometry of the reflecting hyperplanes of $W$. Indeed, most of the results of the paper are proven in the more general setting of simplicial hyperplane arrangements.

Keywords: lattice congruence, noncrossing partition, shard, weak order

\section{Introduction}

The (classical) noncrossing partitions were introduced by Kreweras in [13]. Work of Athanasiadis, Bessis, Biane, Brady, Reiner and Watt [1, 2, 3, 5, 27] led to the recognition that the classical noncrossing partitions are a special case $\left(W=S_{n}\right)$ of a combinatorial construction which yields a noncrossing partition lattice $\mathrm{NC}(W)$ for each finite Coxeter group $W$.

Besides the interesting algebraic combinatorics of the $W$-noncrossing partition lattice, there is a strong motivation for this definition arising from geometric group theory. In that context, $\mathrm{NC}(W)$ is a tool for studying the Artin group associated to $W$. (As an example, the Artin group associated to $S_{n}$ is the braid group.) For the purposes of Artin groups, a key property of $\mathrm{NC}(W)$ is the fact that it is a lattice. This was first proved uniformly (i.e. without a type-by-type check of the classification of finite Coxeter groups) by Brady and Watt [6]. Another proof, for crystallographic $W$, was later given by Ingalls and Thomas [12].

The motivation for the present work is a new construction of $\mathrm{NC}(W)$ leading to a new proof that $\mathrm{NC}(W)$ is a lattice. The usual definition constructs $\mathrm{NC}(W)$ as an interval in a non-lattice (the absolute

\footnotetext{
${ }^{\dagger}$ Partially supported by an FRPD grant from North Carolina State University.

* This is an extended abstract, outlining the results of a paper [24] which will be submitted elsewhere. Some parts of this extended abstract are direct quotes from the complete paper.
}

1365-8050 (C) 2009 Discrete Mathematics and Theoretical Computer Science (DMTCS), Nancy, France 
order) on $W$; we define a new lattice structure $(W, \preceq)$ on all of $W$ and identify a sublattice of $(W, \preceq)$ isomorphic to $\mathrm{NC}(W)$. No part of this construction—other than proving that the sublattice is isomorphic to $\mathrm{NC}(W)$-relies on previously known properties of $\mathrm{NC}(W)$. Thus, one can take the new construction as a definition of $\mathrm{NC}(W)$. The proof that $\mathrm{NC}(W)$ can be embedded as a sublattice of $(W, \preceq)$ draws on nontrivial results about sortable elements established in [21, 22, 25, 26].

Beyond the initial motivation for defining $(W, \preceq)$ - to construct $\mathrm{NC}(W)$ and prove that it is a latticethe lattice $(W, \preceq)$ turns out to have very interesting properties. In particular, many of the properties of $(W, \preceq)$ are precisely analogous to the properties of $\mathrm{NC}(W)$.

The lattice $(W, \preceq)$ is defined in terms of the polyhedral geometry of shards, certain codimension-1 cones introduced and studied in [16, 17, 18, 22]. Shards were used to give a geometric description of lattice congruences of the weak order. In this paper, we consider the collection $\Psi$ of arbitrary intersections of shards, which forms a lattice under reverse containment. Surprisingly, $\Psi$ is in bijection with $W$. The lattice $(W, \preceq)$ is defined to be the partial order induced on $W$, via this bijection, by the lattice $(\Psi, \supseteq)$. Thus we call $(W, \preceq)$ the shard intersection order on $W$.

For the remainder of this extended abstract, we will fill in some additional details about the constructions and results summarized above and in Table 1 . We also illustrate the case $W=S_{4}$.

\section{Shards and intersections of shards}

In this section we define shards and discuss the lattice $(\Psi(W), \supseteq)$, where $\Psi(W)$ is the collection of arbitrary intersections of shards. We then describe a bijection between $\Psi(W)$ and $W$, and use this bijection to define a partial order $(W, \preceq)$ isomorphic to $(\Psi(W), \supseteq)$. The motivation for the definition of shards arises from the study of lattice congruences of the weak order, and will be discussed in Section 5

Finite Coxeter groups correspond to finite reflection groups: finite groups of orthogonal transformations of $\mathbb{R}^{n}$ generated by reflections. Given a finite reflection group $W$, let $T$ be the set of elements of $W$ that act as reflections and let $\mathcal{A}$ be the collection of reflecting hyperplanes of elements of $T$. The set $\mathbb{R}^{n} \backslash(\cup \mathcal{A})$ consists of connected components which are called regions. Each region is an $n$-dimensional simplicial cone. Fixing some region $D$ to represent the identity element, the map $w \mapsto w D$ is a bijection between $W$ and the set of regions.

Example 2.1 As a running example, consider the Coxeter group $W=S_{4}$. This is the group of reflective symmetries of the regular tetrahedron. Exactly six elements of $S_{4}$ act as reflections (the six transpositions). Thus $\mathcal{A}$ consists of six reflecting planes in $\mathbb{R}^{3}$. To visualize this collection of planes, first take the intersection of $\mathcal{A}$ with the unit sphere to obtain a collection of six great circles on the sphere. Then stereographically project the unit sphere to the plane. The great circles map to circles in the plane. The result of this construction appears as Figure $1 \mathrm{a}$. Each of the 24 curvilinear triangles, including the outer triangle, represents a region. Each region is a triangular cone.

The shards are defined ${ }^{(i)}$ as follows: For each hyperplane $H$, we describe a collection of cutting subspaces of $H$. These cutting subspaces are of codimension- 1 in $H$ (and thus of codimension- 2 in the ambient vector space). The shards contained in $H$ are the (closed) regions of this arrangement of codimension- 1 subspaces of $H$. We will say that the cutting subspaces cut $H$ into shards. The complete collection of shards in $\mathcal{A}$ consists of all of the shards in all of the hyperplanes of $\mathcal{A}$. The definition of shards will depend on the choice of $D$, but only up to symmetry.

(i) See [24] Section 3] for a different phrasing of the definition. 
Tab. 1: Properties of $(W, \preceq)$ and $\mathrm{NC}(W)$.

\begin{tabular}{|c|c|}
\hline$(W, \preceq)$ is a lattice. & $\mathrm{NC}(W)$ is a lattice一a sublattice of $(W, \preceq)$. \\
\hline$(W, \preceq)$ is atomic and coatomic. & $\mathrm{NC}(W)$ is atomic and coatomic. \\
\hline $\begin{array}{l}(W, \preceq) \text { is graded, with rank numbers given by the } \\
W \text {-Eulerian numbers. }\end{array}$ & $\begin{array}{l}\mathrm{NC}(W) \text { is graded, with rank numbers given by } \\
\text { the } W \text {-Narayana numbers. }\end{array}$ \\
\hline ( $W, \preceq$ ) is not self-dual. & $\mathrm{NC}(W)$ is self-dual. \\
\hline$(W, \preceq)$ is weaker than weak order. & $\begin{array}{l}\mathrm{NC}(W) \text { is weaker than the Cambrian lattice. } \\
\text { (See [20] for a definition.) }\end{array}$ \\
\hline $\begin{array}{l}\text { Every lower interval }[1, w] \preceq \text { of }(W, \preceq) \text { is isomor- } \\
\text { phic to }\left(W_{J}, \preceq\right) \text { for some standard parabolic sub- } \\
\text { group } W_{J} \text { depending on } w \text {. }\end{array}$ & $\begin{array}{l}\text { Similarly, lower intervals of } \mathrm{NC}(W) \text { are isomor- } \\
\text { phic to noncrossing partition lattices } \mathrm{NC}\left(W_{J}\right) \text {. }\end{array}$ \\
\hline $\begin{array}{l}\text { The Möbius number of }(W, \preceq) \text { is equal, up to a } \\
\text { sign, to the number elements of } W \text { that are not } \\
\text { contained in any proper standard parabolic sub- } \\
\text { group of } W \text {. }\end{array}$ & $\begin{array}{l}\text { The Möbius number of } \mathrm{NC}(W) \text { is equal, up to } \\
\text { a sign, to the number of elements of } \mathrm{NC}(W) \text { that } \\
\text { are not contained in any proper standard parabolic } \\
\text { subgroup of } W \text {. }\end{array}$ \\
\hline $\begin{array}{l}\text { Maximal chains in }(W, \preceq) \text { are in bijection with } \\
\text { maximal simplices in a certain triangulation of } \\
\text { the } W \text {-permutohedron. Loday [14] described the } \\
\text { triangulation in the case } W=S_{n} . \text { The bijection } \\
\text { between maximal chains and maximal simplices } \\
\text { is new for every } W \text {. }\end{array}$ & $\begin{array}{l}\text { A similar bijection holds for } \mathrm{NC}(W) \text { and a tri- } \\
\text { angulation of the } W \text {-associahedron. Loday [14] } \\
\text { described the triangulation and established the } \\
\text { bijection in the case } W=S_{n} \text {. The bijection } \\
\text { between chains and simplices is new for every } \\
\text { other } W \text {. }\end{array}$ \\
\hline $\begin{array}{l}\text { More generally, for each } k \text {, there is a bijection } \\
\text { between } k \text {-chains in }(W, \preceq) \text { and } k \text {-simplices in } \\
\text { the same triangulation of the } W \text {-permutohedron. } \\
\text { This is especially surprising because the triangu- } \\
\text { lation and the order complex of }(W, \preceq) \text { have dif- } \\
\text { ferent topology. This result is new for all } W \text {. }\end{array}$ & $\begin{array}{l}\text { The same is true of } k \text {-chains in } \mathrm{NC}(W) \text { and } k \text { - } \\
\text { simplices in the same triangulation of the } W \text { - } \\
\text { associahedron. This result is also new for all } W \text {. }\end{array}$ \\
\hline $\begin{array}{l}\text { There is a recursion counting maximal chains } \\
\text { in }(W, \preceq) \text { by summing the number of maximal } \\
\text { chains in }\left(W_{J}, \preceq\right) \text { for each maximal proper stan- } \\
\text { dard parabolic subgroup } W_{J} \text {. With }\langle s\rangle=S \backslash\{s\} \text {, } \\
\operatorname{MC}(W, \preceq)=\sum_{s \in S}\left(\frac{|W|}{\left|W_{\langle s\rangle}\right|}-1\right) \operatorname{MC}\left(W_{\langle s\rangle}, \preceq\right) .\end{array}$ & $\begin{array}{l}\text { There is a similar recursion [23, Corollary 3.1] } \\
\text { counting maximal chains in } \mathrm{NC}(W) \text {. } \\
\qquad \mathrm{MC}(\mathrm{NC}(W))=\frac{h}{2} \sum_{s \in S} \mathrm{MC}\left(\mathrm{NC}\left(W_{\langle s\rangle}\right)\right)\end{array}$ \\
\hline
\end{tabular}




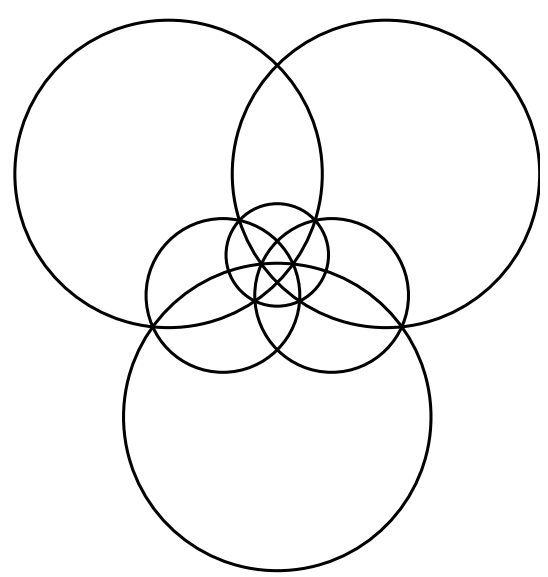

(a)

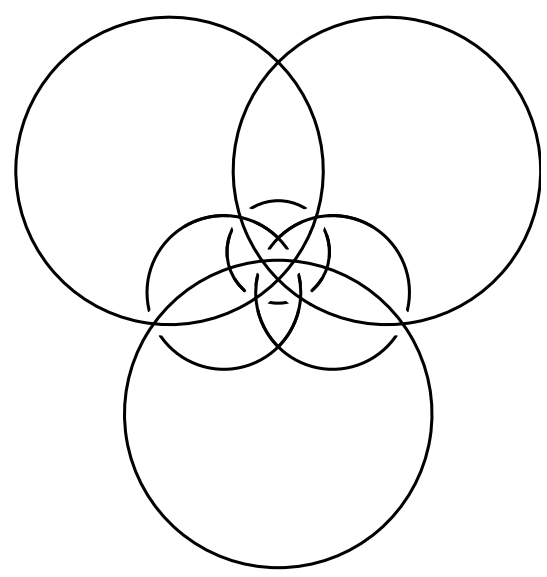

(b)

Fig. 1: a: The reflecting planes of $S_{4}$, in stereographic projection. b: Shards in the case $W=S_{4}$.

Each cutting subspace of $H$ will be the intersection of $H$ with some other hyperplane in $\mathcal{A}$. Given $H$ and $H^{\prime}$ in $\mathcal{A}$, let $\mathcal{A}^{\prime}$ be the set of hyperplanes in $\mathcal{A}$ containing $H \cap H^{\prime}$. Exactly one of the regions defined by $\mathcal{A}^{\prime}$ contains the fixed region $D$; let $D^{\prime}$ denote the $\mathcal{A}^{\prime}$-region containing $D$. The two hyperplanes in $\mathcal{A}^{\prime}$ defining the facets of the cone $D^{\prime}$ are called the basic hyperplanes of $\mathcal{A}^{\prime}$. The subspace $H^{\prime} \cap H$ is a cutting subspace of $H$ if and only if $H$ is not basic in $\mathcal{A}^{\prime}$.

Example 2.2 Figure 2 a illustrates the definition of shards in the case where $W$ is of type $B_{2}$. In this case, for any distinct hyperplanes $H$ and $H^{\prime}$, we have $\mathcal{A}^{\prime}=\mathcal{A}$. Thus the two hyperplanes defining facets of $D$ are never cut, but each of the other hyperplanes is cut at the origin. All of the shards are closed cones containing the origin; however, some shards in the picture are offset slightly to indicate that they do not continue through the origin.

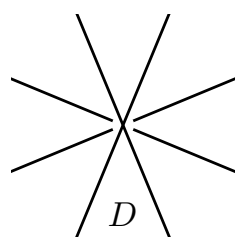

(a)

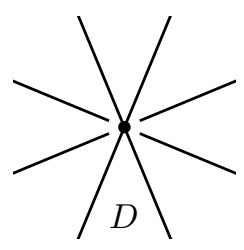

(b)

Fig. 2: (a) Shards in the case $W=B_{2}$. (b) $\Psi\left(B_{2}\right)$.

Example 2.3 The shards, for the case $W=S_{4}$, are pictured in Figure 1 b. This figure is a stereographic projection as explained in Example 2.1. The cone $D$ is the small triangular region which is inside the three largest circles. The shards are closed two-dimensional cones (which in some cases are entire planes). Thus they appear as full circles or as circular arcs in the figure. To clarify the picture, we continue the 
convention of Figure 2 a: Where shards intersect, certain shards are offset slightly from the intersection to indicate that they do not continue through the intersection.

Let $(\Psi(W), \supseteq)$ be the set of arbitrary intersections of shards, partially ordered by reverse containment. It is immediate that $(\Psi(W), \supseteq)$ is a join semilattice; the join operation is intersection. Interpreting the empty intersection of shards to be the ambient vector space, we see that $(\Psi(W), \supseteq)$ is a lattice.

Example 2.4 This example continues Example 2.2. The set $\Psi(W)$, for the case where $W$ is of type $B_{2}$, is pictured in Figure 2 b. The elements of $\Psi(W)$ are closed cones in $\mathbb{R}^{2}$, namely the origin, the six shards, and the whole space $\mathbb{R}^{2}$ (arising as the intersection of the empty set of shards).

Example 2.5 This example continues Example 2.3. The set $\Psi\left(S_{4}\right)$ is pictured in Figure 3 a. The elements of $\Psi\left(S_{4}\right)$ are closed cones in $\mathbb{R}^{3}$, namely the origin, eleven one-dimensional cones (three of which are entire lines), eleven shards (two-dimensional cones, three of which are entire planes) and the whole space $\mathbb{R}^{3}$. Each cone intersects the unit sphere in one of six ways: an empty intersection, a single point, a pair of antipodal points, an arc of a great circle, a great circle, or the entire sphere. Figure 3 a depicts these intersections in a stereographic projection onto the plane. Thus the shards are shown as circles or circular arcs and the one-dimensional cones are pictured as points or pairs of points. A white dot indicates a point which is paired with its antipodal point. (To find antipodal points, note that any two of the circles shown intersect in a pair of antipodal points.)

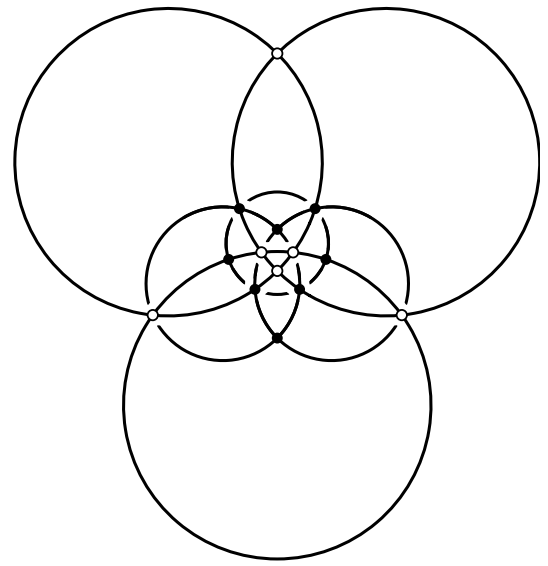

(a)

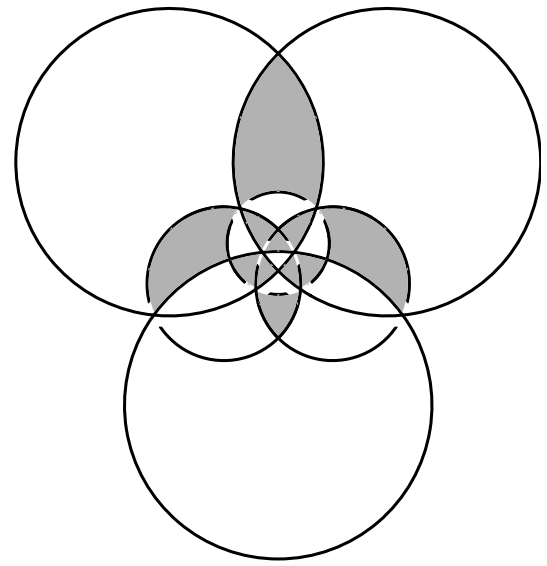

(b)

Fig. 3: (a) $\Psi\left(S_{4}\right)$. (b) Shards and join-irreducible elements in the case $W=S_{4}$.

The most important fact about the set $\Psi(W)$ is that it is in bijection with the elements of the group $W$. The bijection employs the weak order on $W$ and will be explained in Section 3 .

\section{The weak order}

In this section, we review the weak order on a finite Coxeter group. The weak order is a partial order on the elements of a Coxeter group $W$. When $W$ is finite, this partial order is a lattice [4]. The weak order 
is relevant to the present discussion for at least two reasons: to motivate the definition of shards and to explain the bijection between intersections of shards and elements of $W$.

Example 3.1 When $W$ is the symmetric group $S_{n}$, the weak order has a simple description in terms of the one-line notation for permutations: A cover relation in the weak order corresponds to swapping two adjacent entries. Going "up" in the cover relation means placing the two entries out of numerical order. The weak order on $S_{4}$ is illustrated in Figure 4 a.

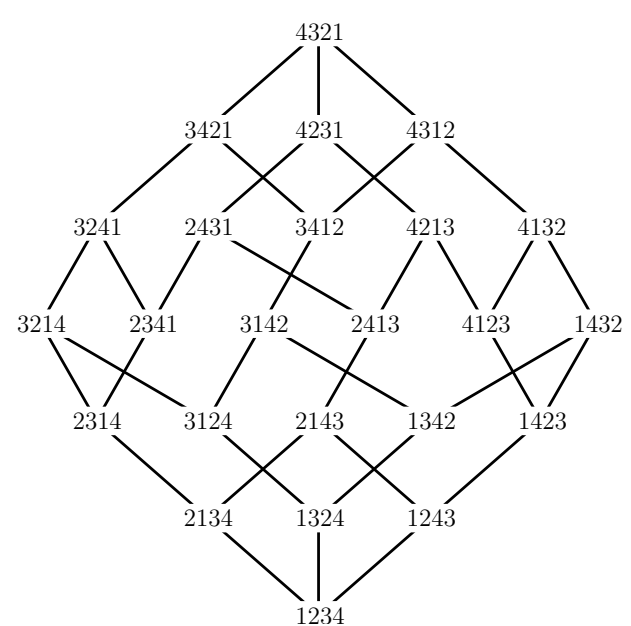

(a)

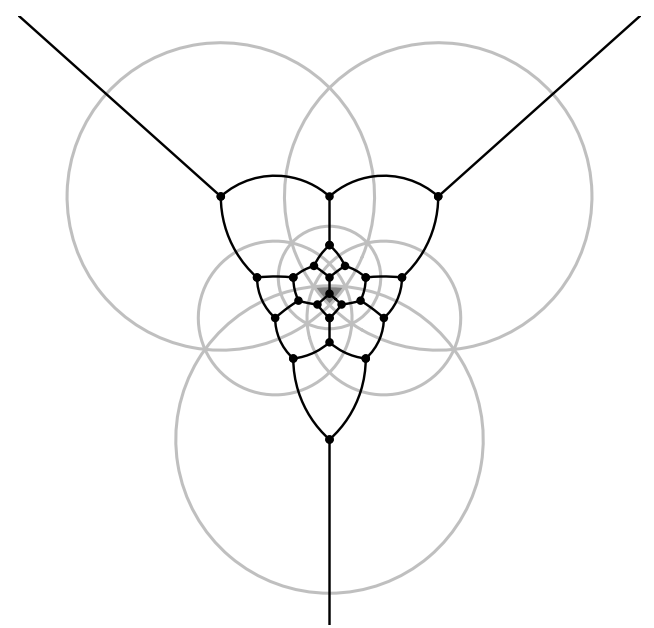

(b)

Fig. 4: Two views of the weak order on $S_{4}$.

The weak order also has a geometric description in terms of the arrangement $\mathcal{A}$ of reflecting hyperplanes. Recall that, once a region $D$ is chosen to represent the identity, the elements of $W$ are in bijection with the regions defined by $\mathcal{A}$. A cover relation in the weak order relates two adjacent regions. If $H$ is the hyperplane separating the two then the lower region is on the same side of $H$ as the identity region $D$.

Example 3.2 The weak order on $W$ can be visualized in the stereographic projection of Figure 1 a. A dot representing each region and an edge representing each cover relation combine to form a "radial Hasse diagram," shown in Figure 4 b. Here the unique minimal element is the central element (contained in the shaded region). The upper vertex of a cover relation is a greater distance in the plane from the center than the lower vertex of the cover. The unique maximal element is the point at infinity.

For general finite $W$, the shards in $\mathcal{A}$ are in bijection [17, Proposition 2.2] with the join-irreducible elements of the weak order: the elements $j \in W$ covering exactly one other element $j_{*} \in W$. The region $j D$ representing $j$ is separated from the region $j_{*} D$ by a common facet of both. The bijection sends $j$ to the unique shard $\Sigma(j)$ containing the common facet. We will write the inverse map as $\Sigma \mapsto j(\Sigma)$.

Example 3.3 This example continues Example 2.3 Figure 3 b again shows the shards for $W=S_{4}$. The shaded triangles correspond to join-irreducible elements. Each such triangle has two convex sides and one 
concave side. The bijection between join-irreducible elements and shards sends the triangle to the shard containing its concave side.

The bijection between join-irreducible elements and shards is the restriction of the bijection between group elements and intersections of shards. We now describe the latter.

Each element $w$ of $W$ has [26, Theorem 8.1] a canonical join representation in the weak order on $W$. A join representation is an expression for $w$ as an irredundant join of join-irreducible elements. The canonical join-representation of $w$ is the unique minimal (i.e. lowest in the partial order) join representation for $W$, in a sense that can be made precise. For finite lattices, the property that each element has a canonical join-representation and a canonical meet representation is equivalent to the property of semi-distributivity [11, Theorem 2.24].

Let $\operatorname{Can}(w)$ be the set of join-irreducible elements occurring in the canonical join representation of $w$. Define a map $\psi: W \rightarrow \Psi(W)$ and a map $\omega: \Psi(W) \rightarrow W$ by setting

$$
\psi(w)=\bigcap_{j \in \operatorname{Can}(w)} \Sigma(j), \quad \text { and } \quad \omega(C)=\bigvee_{\Sigma \supseteq C} j(\Sigma) .
$$

In the latter formula, the sum is over shards $\Sigma$ containing $C$ and the join is taken in the weak order on $W$.

Proposition 3.4 Let $W$ be a finite Coxeter group. Then:

(i) $\psi$ is a bijection from $W$ to $\Psi(W)$ with inverse map $\omega$.

(ii) $\omega$ is an order-preserving map from $(\Psi, \supseteq)$ to the weak order $(W, \leq)$.

(iii) The number of right descents of $w \in W$ equals the codimension of $\psi(w)$.

The right descents of $w$ are the simple generators $s \in S$ such that $\ell(w s)<\ell(w)$. The proof of Proposition 3.4 employs geometric results about the cutting subspaces of hyperplanes as well as lattice-theoretic results about the weak order.

Let $(W, \preceq)$ denote the lattice induced on $W$, via the bijection of Proposition 3.4 from $(\Psi(W), \supseteq)$. We can give a direct characterization of $\preceq$ as follows: Given $x \lessdot y$ in the weak order, let $\Sigma(x \lessdot y)$ be the shard containing the common facet of $x D$ and $y D$. Let $j(x \lessdot y)=j(\Sigma(x \lessdot y))$. Given $w \in W$, define $b(w)$ to be the meet of the elements covered by $w$ and define $A(w)=\{j(x \lessdot y): b(w) \leq x \lessdot y \leq w\}$. Then $v \preceq w$ if and only if $A(v) \subseteq A(w)$. Up to now, the geometric definition of $(W, \preceq)$ has been much more useful in proofs than this direct combinatorial approach.

Example 3.5 Continuing Example 2.5, the lattice $(W, \preceq)$ is shown in Figure 5 a for the case $W=S_{4}$. Readers wishing to work through the details of this example will be aided by [19. Proposition 6.4], where the map $(x \lessdot y) \mapsto j(x \lessdot y)$ is described explicitly in the case $W=S_{n}$.

\section{Properties of $(W, \preceq)$}

In this section, we provide more detail on some of the properties of the lattice $(W, \preceq)$ listed in Table 1 .

Proposition 4.1 The lattice $(W, \preceq)$ is graded, with the rank of $w \in W$ equal to the number of right descents of $w$. Alternately, the rank of a cone $C \in \Psi(W)$ is the codimension of $C$. 


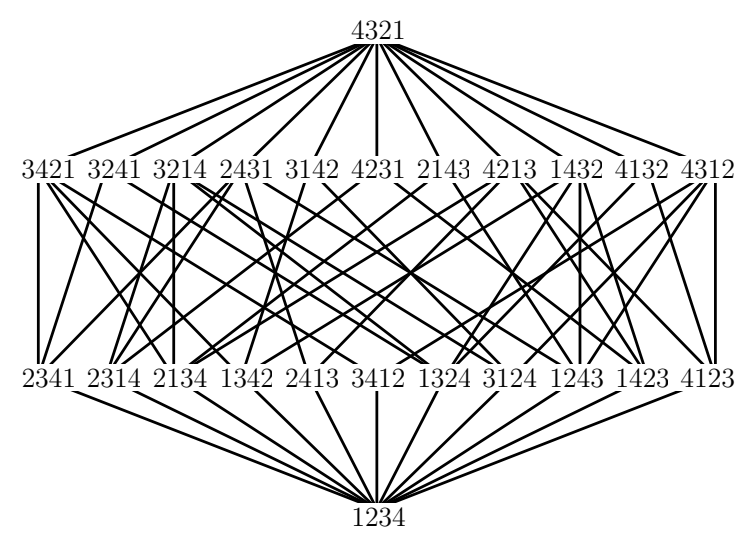

(a)

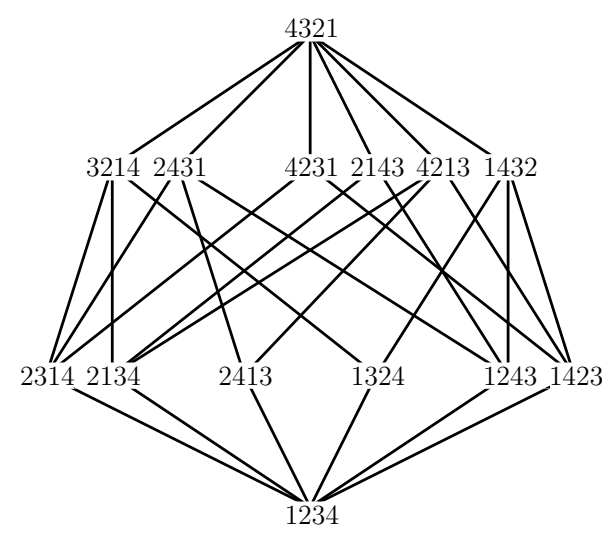

(b)

Fig. 5: (a) $\left(S_{4}, \preceq\right)$. (b) $\left(S_{4}, \preceq\right)$ restricted to $c$-sortable elements.

Theorem 4.2 For any $w \in W$, the lower interval $[1, w] \preceq$ is isomorphic to $\left(W_{J}, \preceq\right)$, where $J=\operatorname{Des}(w)$ is the set of right descents of $w$.

Theorem 4.2 should sound geometrically plausible, once it is translated into the context of $(\Psi(W), \supseteq)$. The lower interval below a cone $C$ in $(\Psi(W), \supseteq)$ is the set of shard intersections containing $C$. This is analogous to the interval below a subspace in the intersection lattice of $\mathcal{A}$. The latter interval is isomorphic to the intersection lattice of $\mathcal{A}_{J}$, the arrangement of reflecting hyperplanes of $W_{J}$, for $J$ as in Theorem 4.2 .

Theorem 4.2 allows us to determine the Möbius number of $(W, \preceq)$ : Up to a sign, it is the number of elements of $W$ not contained in any proper standard parabolic subgroup. The identity element of $W$ is the unique minimal element of $(W, \preceq)$ and the longest element $w_{0}$ is the unique maximal element.

Theorem 4.3 The Möbius function of $(W, \preceq)$ satisfies $\mu_{\preceq}\left(1, w_{0}\right)=\sum_{J \subseteq S}(-1)^{|J|}\left|W_{J}\right|$.

Proof: In light of Theorem 4.2 it is enough to show that the following sum vanishes:

$$
\sum_{w \in W} \sum_{J \subseteq \operatorname{Des}(w)}(-1)^{|J|}\left|W_{J}\right|=\sum_{J \subseteq S}(-1)^{|J|}\left|W_{J}\right| \sum_{\substack{w \in W \\ J \subseteq \operatorname{Des}(w)}} 1 .
$$

The inner sum is the number of maximal-length representatives of cosets of $W_{J}$ in $W$. This number is $|W| /\left|W_{J}\right|$, so the sum reduces to zero.

The fact that the proof of Theorem 4.3 is so simple is an indication that the poset $(W, \preceq)$ is a natural partial order on $W$. Theorem 4.2 also allows us to give a recursive formula for $\operatorname{MC}(W, \preceq)$, the number of maximal chains in $(W, \preceq)$. Recall that for $s \in S$, the symbol $\langle s\rangle$ stands for $S \backslash\{s\}$.

Theorem 4.4 For any finite Coxeter group $W$ with simple generators $S$,

$$
\operatorname{MC}(W, \preceq)=\sum_{s \in S}\left(\frac{|W|}{\left|W_{\langle s\rangle}\right|}-1\right) \operatorname{MC}\left(W_{\langle s\rangle}, \preceq\right) .
$$



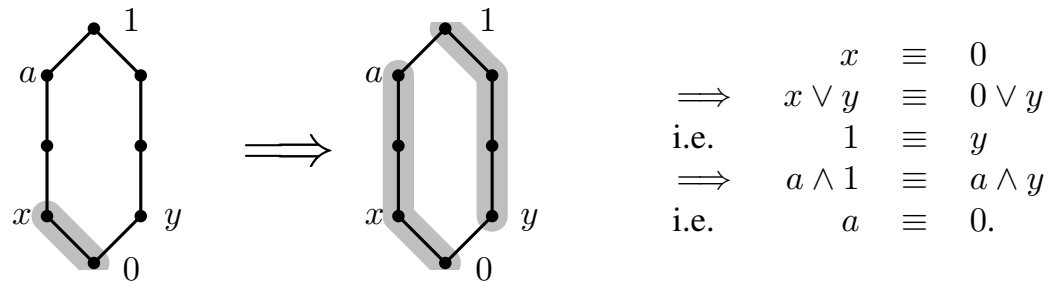

Fig. 6: Forcing in a polygonal lattice.

Proof: The number of maximal chains in $(W, \preceq)$ is the sum over all coatoms $w$ of $(W, \preceq)$ of the number of maximal chains in $[1, w]$. In light of Proposition 4.1, every coatom $w$ is a maximal-length coset representative of the subgroup $W_{\langle s\rangle}$ for some unique $s \in S$. On the other hand, for each $s \in S$, every coset of $W_{\langle s\rangle}$ has a unique maximal-length coset representative. This representative $w$ has $\operatorname{rank}(W)-1$ descents and thus is a coatom of $(W, \preceq)$, except if $w$ is $w_{0}$, which has $\operatorname{rank}(W)$ descents. For each $s \in S$, there are exactly $|W| /\left|W_{\langle s\rangle}\right|$ cosets of $W_{\langle s\rangle}$, and exactly one of these cosets has $w_{0}$ as its maximal length representative. The proposition follows.

Remark 4.5 Recursions involving sums over maximal proper parabolic subgroups (such as the recursion appearing in Theorem 4.4) are very natural in the context of Coxeter groups/root systems. We have seen another such recursion in Table 1 , counting maximal chains in $\mathrm{NC}(W)$. Another example is a recursive formula for the face numbers of generalized associahedra [10, Proposition 3.7] [9, Proposition 8.3]. Yet another is a formula for the volume of the $W$-permutohedron which can be obtained by simple manipulations from Postnikov's formula [15, Theorem 18.3] expressing volume in terms of $\Phi$-trees.

\section{Lattice congruences of the weak order}

In this section, we discuss lattice congruences of the weak order. The goal is to motivate the definition of shards in Section 2 and to lay the groundwork for the discussion of $\mathrm{NC}(W)$ in Section 6

A congruence on a finite lattice $L$ is an equivalence relation $\equiv$ that respects the operations $\vee$ (least upper bound) and $\wedge$ (greatest lower bound). It is easy to verify that congruence classes are always intervals in $L$. Therefore, the relation $\equiv$ is determined by transitivity, once one knows all equivalences of the form $x \equiv y$ for $x \lessdot y$. We say that $\equiv$ squashes the edge $x \lessdot y$ if $x \equiv y$.

Let us consider "building" a congruence by squashing one edge at a time. As one might expect, edges cannot be squashed independently. Rather, there are some forcing relations.

As an example, consider a polygonal lattice $L$. That is, $L$ is composed of two chains of length at least two, with the tops of the two chains identified and the bottoms of the two chains identified.

A "side" edge of the polygon is an edge that is not incident to the top element or the bottom element. A bottom edge is an edge incident to the bottom element, and a top edge is an edge incident to the top element. (Since $L$ is constructed from chains of length at least two, no edge can be both a top edge and a bottom edge.) Edge forcing in a polygonal lattice is described as follows: One easily verifies that side edges can be squashed independently. That is, for any side edge, there is a lattice congruence that squashes that edge an no other edge. In contrast, squashing a bottom edge forces the opposite top edge and all side edges to be squashed, as illustrated in Figure 6 In the figure, squashed edges are highlighted. Dually, squashing a top edge forces the opposite bottom edge and all side edges. 
Many of the intervals in the weak order on $S_{4}$ are polygonal intervals; this is true for the weak order in general. The polygonal intervals are the key to edge-forcings for congruences on the weak order.

Theorem 5.1 Let $W$ be a finite Coxeter group. Then all edge forcings for lattice congruences of the weak order on $W$ are determined locally within polygonal intervals.

For general lattices, forcing is much more complicated. The local property in Theorem 5.1 is equivalent to the assertion that the weak order is congruence normal in the sense of Day [8]. Thus Theorem 5.1 follows from [7, Theorem 6] (Cf. [16, Theorem 27]), where a stronger property, congruence uniformity, is established for the weak order.

Lattice congruences on the weak order have nice geometric properties. Interpret a lattice congruence as an equivalence relation on the regions cut out by the reflecting hyperplanes. For each congruence class $C$, let $\cup C$ denote the union of the regions in $C$. The following is part of [19, Theorem 1.1]

Theorem 5.2 The sets $\cup C$ are the maximal cones of a complete fan.

Shards arise naturally in the context of congruences on the weak order. When we interpret a lattice congruence as an equivalence on regions, and then glue together equivalence classes of regions, squashing edges means removing the common facet (or wall) separating two adjacent regions. A shard is a maximal collections of walls which must always be removed together in a lattice congruence, because of edgeforcing. Edge-forcing also implies some forcing relations among shards. In particular, choosing a lattice congruence on the weak order corresponds to removing a collection of shards that is closed under forcing.

Example 5.3 Consider Example 2.2 and Figure 2 a, and notice that the lattice in Figure 5 is the weak order on $B_{2}$. The discussion above about forcing in polygonal lattices explains why each line bounding the region $D$ is not cut into two shards: a top edge is squashed if and only if the opposite bottom edge is squashed. The other two lines are cut because side edges can be squashed independently. Removing either of the shards bounding $D$ forces each of the four shards not bounding $D$ to be removed also.

\section{Cambrian congruences and $\mathrm{NC}(W)$}

We conclude this extended abstract by returning to the original motivation for the study of $(W, \preceq)$. We review the usual construction of $W$-noncrossing partitions and then explain how the noncrossing partition lattice $\mathrm{NC}(W)$ arises as a sublattice of $(W, \preceq)$.

Let $S$ be the set of simple generators for $W$ and let $T$ be the set of reflections. A Coxeter element $c$ of $W$ is the product, in any order, of the elements of $S$. A reduced $T$-word for $w \in W$ is a shortest possible word for $w$ in the alphabet $T$. (This contrasts with the usual notion of a reduced word for $W$, a shortest possible word for $w$ in the alphabet $S$.) The absolute order on $W$ is the prefix order on reduced $T$-words: we set $u \leq v$ if and only if any reduced $T$-word for $u$ occurs as a prefix of some reduced $T$-word for $v$. The $W$-noncrossing partition lattice $\mathrm{NC}(W)$ is the interval $[1, c]_{T}$ in the absolute order, where $c$ is any Coxeter element of $W$. Up to isomorphism, this definition is independent of the choice of $c$.

Recall that lattice congruences are described by specifying a collection of shards to be "removed." Recall also that there are forcing relations among shards, so that removing one shard may force the removal of another. Let $\Theta$ be a lattice congruence on $W$ and let $\Psi(W / \Theta)$ be the collection of all intersections of shards not removed by $\Theta$. It is immediate that $(\Psi(W / \Theta), \supseteq)$ is a join-sublattice of $(\Psi(W), \supseteq)$.

The noncrossing partition lattice $\mathrm{NC}(W)$ can be realized as $(\Psi(W / \Theta), \supseteq)$ in the case where $\Theta$ is the Cambrian congruence introduced in [20] and studied in [22, 25, 26]. There is a small set $R_{c}$ of shards 
(depending on a choice of Coxeter element $c$ of $W$ ) such that the Cambrian congruence $\Theta_{c}$ corresponds to removing the shards in $R_{c}$ and all other shards whose removal is then forced. The bijection between $W$ and $\Psi(W)$ restricts to an isomorphism between $\left(\Psi\left(W / \Theta_{c}\right), \supseteq\right)$ and the restriction of $(W, \preceq)$ to the $c$ -

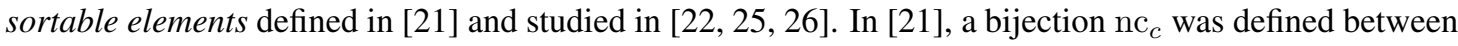
$c$-sortable elements and $\mathrm{NC}_{c}(W)=[1, c]_{T}$. We can now take that result further:

Theorem 6.1 The map $\mathrm{nc}_{c}$ is an isomorphism between the restriction of $(W, \preceq)$ to c-sortable elements and $\mathrm{NC}_{c}(W)$.

As indicated above, the restriction of $(W, \preceq)$ to $c$-sortable elements is isomorphic to the join-sublattice $\left(\Psi\left(W / \Theta_{c}\right), \supseteq\right)$ of $(\Psi(W), \supseteq)$. In fact, one can show that $\left(\Psi\left(W / \Theta_{c}\right), \supseteq\right)$ is a sublattice of $(\Psi(W), \supseteq)$.

Corollary 6.2 The poset $\mathrm{NC}_{c}(W)$ is a lattice.

The first uniform proof [6] that $\mathrm{NC}_{c}(W)$ is a lattice also used the polyhedral geometry of cones. That proof is, in a sense, dual to the proof discussed here (in the broadest outlines but not in any of the details).

Example 6.3 Figure 5 b shows the restriction of $\left(S_{4}, \preceq\right)$ to $c$-sortable elements, with $c=\left(\begin{array}{ll}1 & 2\end{array}\right)(34)(23)$.

\section{Acknowledgments}

This research was greatly assisted by Stembridge's maple packages coxeter/weyl and posets, and by Sloane's On-Line Encyclopedia of Integer Sequences.

\section{References}

[1] C. A. Athanasiadis and V. Reiner, Noncrossing partitions for the group $D_{n}$. SIAM J. Discrete Math. 18 (2004), no. 2, 397-417.

[2] D. Bessis, The dual braid monoid. Ann. Sci. École Norm. Sup. (4) 36 (2003), no. 5, 647-683.

[3] P. Biane, Some properties of crossings and partitions. Discrete Math. 175 (1997), no. 1-3, 41-53.

[4] A. Björner, Orderings of Coxeter groups. Combinatorics and algebra (Boulder, Colo., 1983), 175195, Contemp. Math., 34, Amer. Math. Soc., Providence, RI, 1984.

[5] T. Brady and C. Watt, $K(\pi, 1)$ 's for Artin groups of finite type. Proceedings of the Conference on Geometric and Combinatorial Group Theory (Haifa, 2000). Geom. Dedicata 94 (2002), 225-250.

[6] T. Brady and C. Watt, Non-crossing partition lattices in finite real reflection groups. Trans. Amer. Math. Soc. 360 (2008), 1983-2005.

[7] N. Caspard, C. Le Conte de Poly-Barbut and M. Morvan, Cayley lattices of finite Coxeter groups are bounded. Adv. in Appl. Math. 33 (2004), no. 1, 71-94.

[8] A. Day, Splitting lattices generate all lattices. Algebra Universalis 7 (1977), no. 2, 163-169.

[9] S. Fomin and N. Reading, Generalized cluster complexes and Coxeter combinatorics. Int. Math. Res. Not. 2005, no. 44, 2709-2757. 
[10] S. Fomin and A. Zelevinsky, $Y$-systems and generalized associahedra. Ann. of Math. (2) 158 (2003), no. 3, 977-1018.

[11] R. Freese, J. Ježek, and J. Nation, Free lattices. Mathematical Surveys and Monographs 42. American Mathematical Society, Providence, RI, 1995.

[12] C. Ingalls and H. Thomas, Noncrossing partitions and representations of quivers. preprint, 2006 (math.RT/0612219).

[13] G. Kreweras, Sur les partitions non croisées d'un cycle. Discrete Math. 1 (1972), no. 4, 333-350.

[14] J.-L. Loday, Parking functions and triangulation of the associahedron. Contemp. Math. 431, $327-$ 340. Amer. Math. Soc., Providence, RI, 2007.

[15] A. Postnikov, Permutohedra, associahedra and beyond. preprint, 2005 (arXiv: math / 0507163 ).

[16] N. Reading, Lattice and order properties of the poset of regions in a hyperplane arrangement. Algebra Universalis 50 (2003), 179-205.

[17] N. Reading, The order dimension of the poset of regions in a hyperplane arrangement. J. Combin. Theory Ser. A 104 (2003) no. 2, 265-285.

[18] N. Reading, Lattice congruences of the weak order. Order 21 (2004) no. 4, 315-344.

[19] N. Reading, Lattice congruences, fans and Hopf algebras. J. Combin. Theory Ser. A 110 (2005) no. 2, 237-273.

[20] N. Reading, Cambrian Lattices. Adv. Math. 205 (2006), no. 2, 313-353.

[21] N. Reading, Clusters, Coxeter-sortable elements and noncrossing partitions. Trans. Amer. Math. Soc. 359 (2007), no. 12, 5931-5958.

[22] N. Reading, Sortable elements and Cambrian lattices. Algebra Universalis 56 (2007), no. 3-4, 411437.

[23] N. Reading, Chains in the noncrossing partition lattice. SIAM J. Discrete Math. 22 (2008), no. 3, 875-886.

[24] N. Reading, Noncrossing partitions and the shard intersection order. Preprint, 2009.

[25] N. Reading and D. Speyer, Cambrian Fans (math. CO/0606201). J. Eur. Math. Soc. (JEMS), to appear.

[26] N. Reading and D. Speyer, Sortable elements in infinite Coxeter groups (arXiv:0803.2722). preprint, 2008.

[27] V. Reiner, Non-crossing partitions for classical reflection groups, Discrete Math. 177 (1997), no. 13, 195-222. 